\title{
Nonuniform Rational Basis Spline Interpolation for Off-Axis Aspheric Mirror Grinding Based on Wheel Path Planning
}

\author{
Jianpu Xi $\mathbb{D}^{1},{ }^{1}$ Laibang Zhang $\mathbb{D}^{1},{ }^{1}$ Qing Yang $\mathbb{D}^{2},{ }^{2}$ Bin $\mathrm{Li},{ }^{1}$ Zexiang Zhao $\mathbb{D}^{1},{ }^{1}$ \\ and Cong Zhang $\mathbb{D}^{1}$ \\ ${ }^{1}$ School of Mechatronics Engineering, Zhongyuan University of Technology, Zhengzhou 450007, China \\ ${ }^{2}$ Center for Modern Educational Technology, Zhongyuan University of Technology, Zhengzhou 450007, China \\ Correspondence should be addressed to Jianpu Xi; xjpyq2010@zut.edu.cn
}

Received 4 November 2020; Revised 10 February 2021; Accepted 3 March 2021; Published 26 March 2021

Academic Editor: Yuanchang Liu

Copyright (c) 2021 Jianpu Xi et al. This is an open access article distributed under the Creative Commons Attribution License, which permits unrestricted use, distribution, and reproduction in any medium, provided the original work is properly cited.

Large-diameter aspheric mirrors made of hard and brittle materials suffer from low efficiency in the grinding stage, making them difficult to process. In this study, a nonuniform rational basis spline (NURBS) interpolation method is proposed and developed based on the grinding wheel path planning. The characteristic parameters of NURBS machining program are calculated to ensure the accuracy of curve interpolation path planning, and a series of control points are selected according to the normal vector points of the grinding wheel path. The control points were determined by the distance between the aspheric tangent and the next type value point at a fixed distance $d$. We then established a mathematical model for the knot point and simulated the relationship between the knot point and $d$. This indicated that $d$ must be optimized according to the number of points to reduce the number of control points. According to this established model, a comparison experiment was conducted using an off-axis aspheric mirror, with a $280 \mathrm{~mm}$ diameter. Straight line and NURBS grinding were compared, and the NURBS interpolation method was shown to effectively improve grinding accuracy and efficiency by about $30 \%$.

\section{Introduction}

Scientific and technological development has resulted in aspheric optical components being utilized widely, as large and medium optical aspheric surfaces play an increasingly important role in various types of instruments and equipment including astronomical telescopes, laser fusion devices, interferometers, lenses in lithography, and other apparatus. Correspondingly, manufacturing requirements have become increasingly strict. Specifically, off-axis aspheric mirrors that are key components in laser systems face stringent manufacturing standards with increasing popularity and demand [1]. These components are made of hard and brittle materials that require grinding during processing. Due to the long machining time and high surface accuracy requirement, this grinding stage becomes a bottleneck and limits the whole processing cycle. Further, the grinding process is prone to introducing surface profile errors and subsurface damage, due to the particulate nature of the raw materials, which negatively affect the subsequent polishing stage performance. Because of the structural characteristics of the off-axis aspheric mirror, its machining process is more complicated than a symmetric aspheric mirror. It is very important to improve machining efficiency and accuracy. Much research has been done on freeform surface fabrication techniques, including aspherical reflectors [2]. Sarma et al. studied tool path trajectory generation for computerised numerical control (CNC) grinding and proposed a comprehensive method to consider trajectory generation for both manufacturing and measurement [3]. Lee et al. used the Form Talysurf system to modify the CNC machine tool code that generated the ultraprecision single-point diamond turning machine tool path and effectively compensated for the residual form error caused by decentralising the tool during the ultraprecision machining process [4]. To eliminate the influence of residual errors on machining accuracy, Stephen studied tool path generation methods for multiaxis CNC machining of freeform surfaces, proposed a minimum time tool path, and discussed local and global tool path generation [5]. Based on the analysis of aspheric lens array, a 
method for generating diamond forming track based on tool radius compensation in the three-dimensional path is proposed. A single crystal diamond tool for precise microaspherical lens array on four-axis precision machine tool has been developed by means of path generation, which improves the machining accuracy [6].

Processing nonspherical mirrors primarily utilizes cross or parallel grinding modes, and the processing program is generated according to the desired geometric parameters. Huang et al. reduced the influence of grinding wheel geometric errors on aspheric profile accuracy by setting an initial grinding cycle. They then proposed that the next grinding cycle be measured and modified by the Talysurf profiler along the grinding point. This method corrected the tool path, using the residual error in the normal direction, to obtain a more accurate path [7]. By studying the influence of wheel wear on grinding profile error, Hwang et al. proposed a new wheel radius equation that easily derives processing parameters. Through relevant experimental analysis and comparison, the wheel radius error is concluded to be primarily affected by the vertical deviation between the wheel rotation centre and the true centre and is effectively consistent with the simulated value [8].

As the applications of aspheric surfaces expand and broaden, the demand for off-axis aspheric surfaces increases. Therefore, many researchers have focused on offaxis aspheric components. In the early $1990 \mathrm{~s}$, British scholar William designed and manufactured a large-scale three-axis CNC machine tool because traditional optical manufacturing methods were unsuitable for off-axis aspheric surfaces. A combination of laser interferometer systems has been used to realize the processing of off-axis aspheric mirrors [9]. Guo et al. proposed a new parallel grinding method using circular diamond grinding wheels, which addressed the inflexibility of nonaxisymmetric aspheric lenses in the mould grinding process and the subsequent need for high precision when grinding spherical diamond wheels in the CNC machining centre. This method was proven, through grinding experiments, to meet the requirements of high precision nonaxisymmetric aspheric lens grinding [10]. It is noteworthy that if the tool path is not smooth during machining, large acceleration changes will lead to subsurface damage, which will affect the quality of surface processing. Therefore, a smooth grinding path should be used when processing aspheric surfaces to reduce component acceleration and deceleration and thereby reduce the impact forces and corresponding subsurface damage. Hence, the traditional grinding method for aspheric components utilises a large amount of small straight segments to approach the parameter curve. The discrete tool path is composed of standard CNC codes G01 as output for linear interpolation, which is prone to errors and difficult to optimise for speed [11-14]. The current nonuniform rational basis spline (NURBS) interpolation algorithm has been applied to aspheric grinding to produce high-precision aspheric components. Specifically, Comley proposed parallel grinding for large-diameter aspheric off-axis aspheric surfaces and used a box structure to grind the inclined cup wheel of the machine tool to achieve $X Z$ plane and $\theta$ axis interpolation grinding that resulted in high-precision lenses [15]. Additionally, Park approximated each type of aspheric NURBS surface within the specified accuracy range and used the corresponding NURBS curves to reconstruct surfaces during aspheric manufacturing, thus proving this method effective [16].

In summary, NURBS interpolation has been widely used in the fields of turning and grinding, but little research has been done on the relationship between grinding efficiency and accuracy. According to practical project requirements, this study proposes a NURBS interpolation method for off-axis aspheric grinding that is based on the grinding wheel path planning. By analysing the characteristic parameters of the NURBS curve, the distance $d$ between the aspheric equation curve tangent and the next type value point was used to determine a series of control points, establish a mathematical model for the type value point, and obtain a simplified curve interpolation that meets all accuracy requirements. The path planning scheme reduces the number of control points on the processing path, improves the processing efficiency and accuracy, and verifies the rationality and practicability of the interpolation algorithm. It provides a reliable and effective theoretical basis and path optimization scheme for grinding nonspherical mirror parts.

\section{Path Planning for Off-Axis Aspheric Grinding}

2.1. Off-Axis Aspheric Equation. As large-scale aspheric mirrors developed and became more sophisticated, they can be classified into symmetric and off-axis aspheric surfaces. In general, the axisymmetric aspheric surface is also known as the surface of rotation, and its equation is based on that of a quadric surface [17].

Off-axis aspheric mirrors are now increasingly used in laser fusion, optical instruments, astronomical cameras, and other related fields. The equation for an off-axis aspheric mirror can be defined as a part of the rotating symmetric aspheric surface [18]:

$$
y(x, z)=-R_{x}+\sqrt{R_{x}^{2}-x^{2}}+\frac{c z^{2}}{1+\sqrt{1-(1+k) c^{2} z^{2}}}
$$

where $R_{x}$ is the base radius of the aspheric principal axis and $c$ is the radius of the aspheric subaxis. Notably, this surface exhibits different shapes and characteristics when employed for different purposes, including off-axis eccentric aspheric mirrors and off-axis wedge aspheric mirrors.

2.2. Off-Axis Aspheric Grinding Process. Manufacturing techniques for off-axis aspheric mirrors include grinding and forming, precision grinding, and precision polishing. At the grinding and forming stage, the rough portion is first ground and formed and then further processed by polishing according to the desired surface profile. Symmetrical aspheric mirror processing can be completed using $X Z C$ machine tool three-axis coordination during grinding. 
However, large-scale off-axis aspheric mirrors adopt a rotary grinding method, which generates the empty tool phenomenon, thus increasing equipment stroke and reducing processing efficiency. Chen et al. proposed adjusting the inclination angle in a precision $\mathrm{CNC}$ grinding system to process off-axis wedge-shaped aspheric mirrors. Alternatively, Guo used the envelope method to plan and process the off-axis aspheric mirror. To avoid the empty tool phenomenon, grinding processes for off-axis aspheric surfaces utilise grating path planning, which can achieve high-precision grinding. Additionally, three-axis equipment is used to grind off-axis aspheric surfaces through parallel grinding, where the linear motion of the grinding wheel is parallel to the direction of the workpiece movement [10]. Compared with the cross grinding of a symmetric aspheric mirror, this method causes the wheel to wear significantly and with symmetry, so that the wheel grinding point must only move along a certain generatrix to grind the aspheric surface. During the machining process, the arc surface of the grinding wheel contact point does not change, and the grinding wheel must therefore be corrected regularly. The off-axis square aspheric grating processing path planning is shown in Figure 1, where the red surface represents the 3D processing path and the blue plane represents the $2 \mathrm{D}$ top view, where the simulated processing data points were generated by linear interpolation. This interpolation was typically done using small steps, so that the processing points are dense and a high-quality profile could be obtained. Specifically, there are usually up to 30,000 processing points on the path, significantly increasing the grinding required to process these surfaces.

Because there are many linear interpolation processing blocks, the linear motion axis acceleration changes frequently, with a particularly large acceleration at the connection to the linear segment. This causes machine tool vibration and accelerated wear and negatively affects the accuracy of the workpiece surface profile. A NURBS interpolation algorithm is commonly used to address these shortcomings of traditional curve interpolation methods. The NC program code for NURBS interpolation is generated based on the power and given values of the control point, weight factor, node vector, and basis function. Therefore, the number of the processing code is only traditional $10 \%$, which greatly reduces processing time and improves machining accuracy.

\section{Optimizing the NURBS Curve Machining Path}

3.1. NURBS Curve Interpolation. The NURBS curve interpolation method was applied to a three-axis combined grinder to improve its machining efficiency and accuracy, as NURBS provides a special mathematical algorithm that reduces the required number of surface feature points. We assume a familiarity with the concept of B-splines as a basis for the following discussion, but the detailed background can be found in [19]. A NURBS curve of the order $($ degree +1$)$, $p$, can be defined by

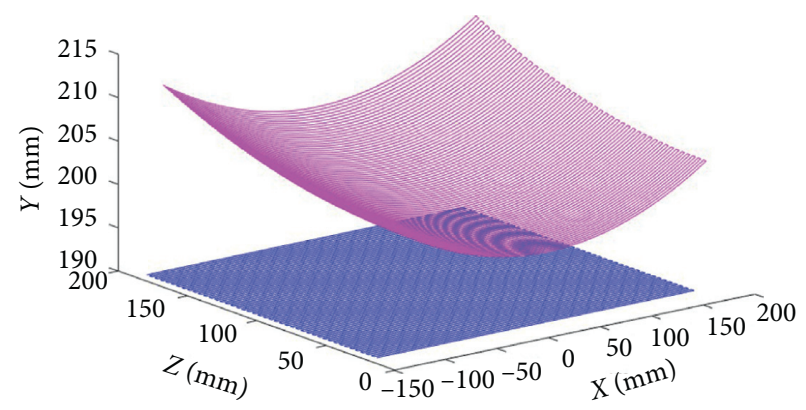

FIGURE 1: The grating path of off-axis square aspheric.

$$
C(u)=\frac{\sum_{i=0}^{n} N_{i, k}(u) w_{i} P_{i}}{\sum_{i=0}^{n} N_{i, k}(u) w_{i}}
$$

where $P_{i}$ are control points, $N_{i, k}(u)$ are normalized B-spline functions of order $p$ defined on the knot vector $T=\left\{t_{0}\right.$, $\left.t_{0}, \ldots t_{\mathrm{n}+\mathrm{p}-1}, t_{\mathrm{n}+\mathrm{p}}\right\}$, and $w_{i}$ are weights. $N_{i, 0}(u)$ is defined as follows:

$$
N_{i, 0}(u)= \begin{cases}1, & u_{i} \leq u \leq u_{i+1}, \\ N_{i, k}(u)=\frac{u-u_{i}}{u_{i+k}-u_{i}} N_{i, k-1}(u) & \\ +\frac{u_{i+k+1}-u}{u_{i+k+1}-u_{i+1}} N_{i+1, k-1}(u), & \\ 0, & i=0,1, \ldots, n .\end{cases}
$$

The curve interpolation method described here adopts the time series $t_{i}(i=1,2, \ldots, n)$, and parameter variables are subdivided into the parameter sequence $u_{i}$ according to this time series to obtain the processing point $C\left(u_{i}\right)$. The control points, weights, and node vector can accurately describe the grinding curve, and the shape of the curve is controlled by specifying and adjusting the control points. Taking these three parameters as part of the NC machining program and generating NURBS curves within the system, novel interpolation machining can be achieved.

3.2. Optimizing the Machining Path Control Points for OffAxis Aspheric Surfaces. The off-axis aspheric grinding process here utilizes the NURBS interpolation method. First, the ordered discrete grinding wheel motion path points must be determined. NURBS curve fitting helps us to obtain the appropriate control points and knot vector parameters so that the tool path can achieve the required processing performance in the grinding stage. It is important to keep in mind that off-axis aspheric grinding differs from general grinding processes. Specifically, during grinding, the contact points of the grinding wheel vary with the position of the grinding wheel. Therefore, the position coordinates of the centre of the grinding wheel must be calculated according to the characteristics of the grinding wheel. Discrete surface 
points are obtained from the ideal parameter equation of the off-axis aspherical surface. Because the curvature of the large-scale aspheric surface does not change significantly, the grinding path of envelope machining is the same as that of grating machining. The coordinates of the grinding point $H$ are $H\left(x_{t}, y_{t}, z_{t}\right)$. Because the grinding curved surface is aspheric, the grinding wheel centre coordinates must be obtained by the normal vector, which is recorded as $n(a, b$, $c$ ). The relative centre of the grinding wheel and the grinding point position during the grinding process are shown in Figure 2. The coordinates of the grinding wheel centreline can then be obtained from the grinding point as $O$ $\left(x_{w}, y_{w}, z_{w}\right)$, so the machining path of the grinding wheel, according to the normal vector, can be expressed as follows:

$$
\left[\begin{array}{c}
x_{w} \\
y_{w} \\
z_{w}
\end{array}\right]=\left[\begin{array}{c}
x_{t} \\
y_{t} \\
z_{t}
\end{array}\right]+(R+r)\left[\begin{array}{c}
\frac{a}{\sqrt{a^{2}+b^{2}}} \\
\frac{b}{\sqrt{a^{2}+b^{2}}} \\
\frac{c}{\sqrt{a^{2}+b^{2}}}
\end{array}\right]
$$

where $R$ is the basic radius of the grinding wheel and $r$ is the radius of the grinding wheel arc.

For off-axis aspheric surfaces, according to the envelope geometry of the grinding wheel arc, the front arc of the wheel changes with the processing position; hence, the centre point of the grinding wheel normal vector is defined as follows:

$$
\left[\begin{array}{l}
x_{w} \\
y_{w} \\
z_{w}
\end{array}\right]=\left[\begin{array}{c}
x_{t} \\
y_{t} \\
z_{t}
\end{array}\right]+r\left[\begin{array}{c}
\frac{a}{\sqrt{a^{2}+b^{2}}} \\
\frac{b}{\sqrt{a^{2}+b^{2}}} \\
\frac{c}{\sqrt{a^{2}+b^{2}}}
\end{array}\right]+R\left[\begin{array}{c}
\frac{a}{\sqrt{a^{2}+b^{2}+c^{2}}} \\
\frac{b}{\sqrt{a^{2}+b^{2}+c^{2}}} \\
0
\end{array}\right] .
$$

Then, the spatial trajectory of the grinding wheel can be obtained according to its grating trajectory. The aforementioned interpolation point can be used as the type value point of the NURBS curve fitting, and the NURBS curve at this type value point can be obtained.

3.3. Machining Path NURBS Curve Fitting. Here, the leastsquares method is used to fit the NURBS curve without passing through each point of the grinding wheel trajectory. The error and minimum of each grinding point on the fitting curve are achieved by selecting the appropriate control point and position. During fitting, the NURBS interpolation path characteristic parameters must be calculated according to the discrete value points on the grinding path. In this study, the simulated aspheric mirror had an error $d$ of $0.01 \mathrm{~mm}$ and obtained 46 control points. The distance between these points in the $X$ direction was about $6.23 \mathrm{~mm}$. The NURBS

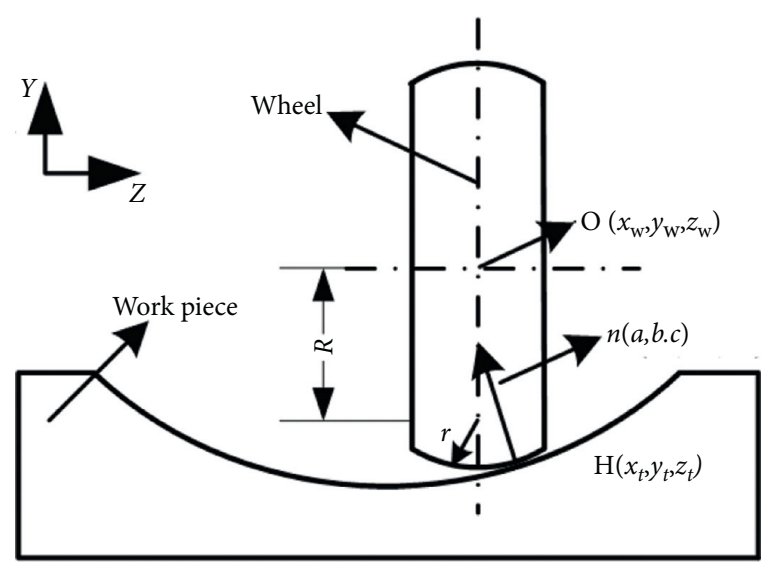

FIGURE 2: The grinding point for off-axis aspheric.

fitting curve was then determined using these generated control points.

In the $O X Y$ coordinate system, the aspheric surface parameter equation is a quadratic surface equation. To better fit the aspheric curve, discrete value points were found using connections between the coordinate points of the grinding wheel path, the minimum principle, the distance between the midpoint of the line between the two type value points and the curve. However, the intensive movement value points, obtained by ordinary linear interpolation, cause the NURBS curve fitting efficiency to be low, as the excessive amount of data yields too many control points, which directly and negatively affects the processing efficiency. To address this, a tangent error control method is proposed. As shown in Figure 3, the starting point for grinding the off-axis aspheric surface is set to $Q_{1}$. A tangent line $L_{1}$ is then drawn at $Q_{1}$, and the next point $Q_{2}$ is found by searching in the positive direction along the tangent $X$-axis. The distance $d$ between the curve and tangent is required to satisfy the accuracy requirement. The distance is defined as the value of the control error factor, and the distance from the tangent point of the aspheric surface to the point next to the tangent line is a fixed value. Thus, the same process can be followed, drawing a tangent line at $Q_{2}$ and searching for the next point that has a distance $d$, equal to the control error, to the tangent line. Then, $n$ type value points can be determined, thus revealing the fitting characteristic data point set $Q$. This set effectively compresses the fitting control vertex of the aspheric NURBS curve.

When $z$ is the determined cross-sectional value $z_{e}$, a path curve for raster interpolation can be expressed as follows:

$$
y(x)=-R_{x}+\sqrt{R_{x}^{2}-x^{2}}+\frac{z_{e}^{2} c}{1+\sqrt{1-(1+k) c^{2} z_{e}^{2}}} .
$$

Using this as the slope of the tangent line at $\left(x_{n}, y_{n}\right)$, the equation of the tangent line becomes

$$
y=-\frac{x_{n}}{\sqrt{R_{x}^{2}-x_{n}^{2}}} x+\frac{x_{n}^{2}}{\sqrt{R_{x}^{2}-x_{n}^{2}}}-R_{x}+\sqrt{R_{x}^{2}-x_{n}^{2}} .
$$




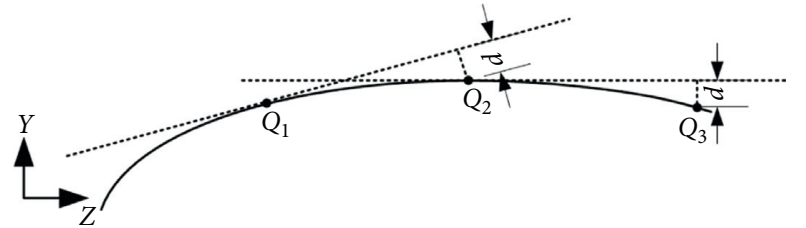

FIGURE 3: Schematic of the fitting process for the characteristic data set $Q$.

If the distance on the tangent line between the point $\left(x_{n+1}, y_{n+1}\right)$ on the curve and the crossing point $\left(x_{n}, y_{n}\right)$ is $d$, the calculation equation is as follows:

$$
d=\left|\frac{-x_{n} x_{n+1}-\sqrt{\left(R_{x}^{2}-x_{n+1}^{2}\right)\left(R_{x}^{2}-x_{n}^{2}\right)}+R_{x}^{2}}{R_{x}}\right| .
$$

The next coordinate point, $x_{n+1}$, is obtained using the distance between the point and the straight line where $d$ is a custom error and $x_{n}$ is a known point. Thus, the coordinate value $x_{n+1}$ for the next point is obtained. In this way, the coordinate points $\left(x_{n+1}, y_{n+1}\right)$ of all points on the curve, and thereby the control vertex for fitting, can be determined.

Assuming the base radius of the aspheric surface to be $R_{x}=2000 \mathrm{~mm}$, the relationship between the intercoordinate distance $L$ and $d$ can be estimated as shown in Figure 4. Here, $L$ changes nonlinearly as the error value $d$ increases. As the figure demonstrates, $d$ values between 0.01 and $0.04 \mathrm{~mm}$ generate $X$ coordinate distances between 4 and $12 \mathrm{~mm}$, which will greatly reduce the number of control vertices and subsequently improve the fitting accuracy. For the same error $d$ range, increasing the aspheric major axis radius $r$ corresponds to wider spacing for $L$, indicating that the curvature radius affects the spacing selection. In this case, the distance is reduced to $0-0.01 \mathrm{~mm}$, and the generated vertex spacing is $0-3 \mathrm{~mm}$. Therefore, the number of vertices for large-diameter aspheric processing is relatively dense, and the grinding efficiency cannot be improved in this manner. Therefore, errors need to be calculated according to the size of the aspheric surface. For accuracy and efficiency, the value of error $d$ must be selected.

With the selection of $d$, the type value points can be obtained enabling the NURBS curve to be fit and the characteristic parameters to be calculated. Hence, these type value points are used as characteristic points. To fit the NURBS curve, the weight factor must first be determined. This factor represents the influence of each control point on the shape of the curve. In general engineering calculations, the weight factor is $w_{i}=1, i=0,1, \ldots, l$. Since the B-spline basis function satisfies:

$$
\sum_{i=0}^{l} N_{i, p}(u)=1
$$

the NURBS curve fitting can be written simply as follows:

$$
C(u)=\sum_{i=0}^{l} N_{i, p}(u) P_{i}
$$

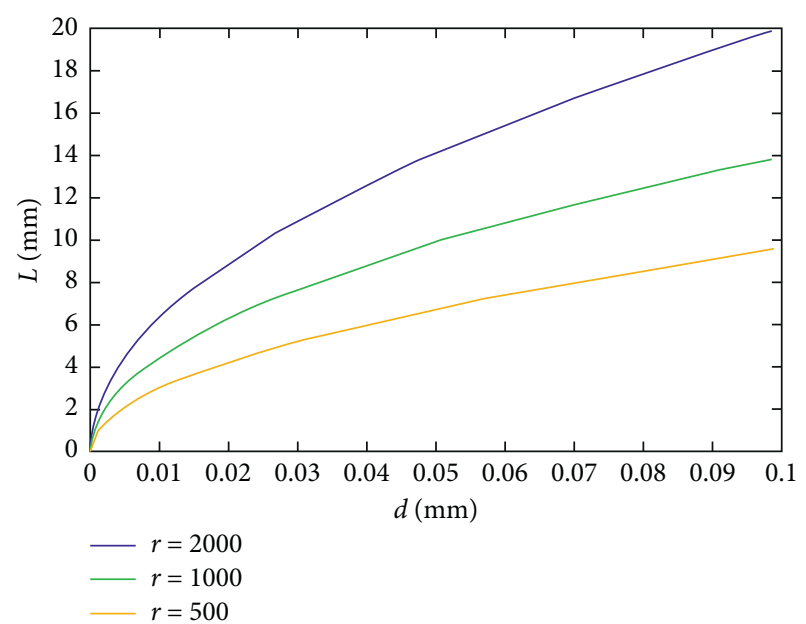

FIGURE 4: Relationship between the intercoordinate distance $(L)$ and the parameter $(d)$ for varied curvature radii $(r)$.

The grinding point $Q_{2}$ of the grinding wheel corresponds to points on the fitting NURBS curve, where

$$
Q_{j}=C\left(u_{j}\right)=\sum_{i=0}^{l} N_{i, p}\left(u_{j}\right) P_{i} .
$$

In the aforementioned formula, $U_{j}$ is the value of this corresponding parameter. According to the principle of least squares approximation, $Q_{j}$ approximation at other points is the optimized objective function:

$$
E\left(R_{0}, R_{1}, \ldots, R_{l}\right)=\sum_{j=0}^{n}\left(C\left(u_{j}\right)-Q_{j}\right)^{2} \longrightarrow \min (J) .
$$

If the total deviation is minimised, partial derivatives at the qth unknown control points must all be equal to zero:

$$
\frac{\partial E}{\partial R_{q}}=2 \sum_{j=0}^{n}\left[N_{q, p}\left(u_{j}\right) \cdot\left(\sum_{i=0}^{l} N_{i, p}\left(u_{j}\right) P_{i}-Q_{j}\right)\right]^{2}=0 .
$$

Thus, a simplified equation can be obtained as follows:

$$
\sum_{j=0}^{n} N_{q, p}\left(u_{j}\right) \sum_{i=0}^{l} N_{i, p}\left(u_{j}\right) P_{i}=N_{q, p}\left(u_{j}\right) Q_{j} .
$$

By solving the aforementioned equation, the control points, and thus the NURBS curve equation fitting according to the type value points, can be obtained. The fitting error is calculated based on the fitting curve to determine the rationality of the control point selection.

The NURBS curve is fitted using the least-squares method described above, and the results are shown in Figure 5. Notably, the NURBS fitting curve does not pass the control points. The fitted curve varies with the curvature of the control vertex connection line. Furthermore, we can ascertain whether the accuracy requirement is met using the vertical distance between the control vertex and the fitted 


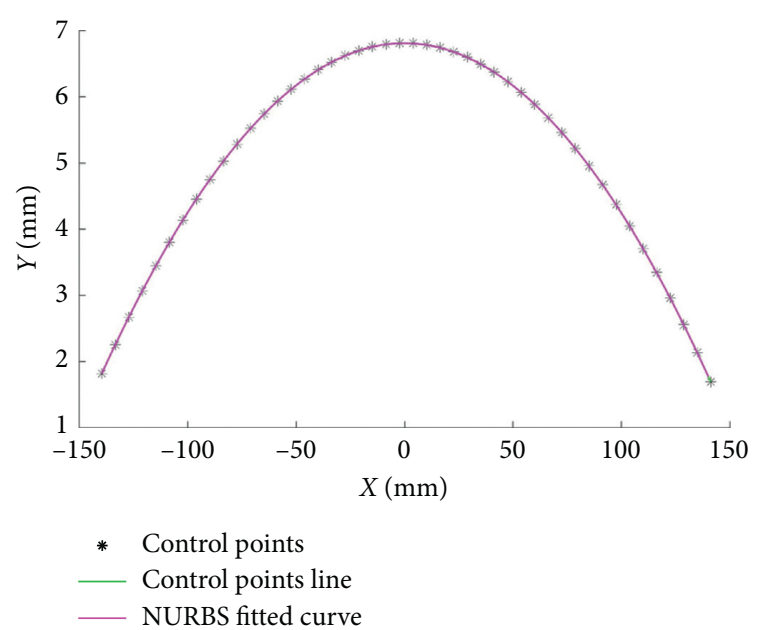

FIgURE 5: The NURBS fitting curve of control points.

curve. The simulation results indicate that the surface profile error is $2-3 \mu \mathrm{m}$, which meets the requirements for aspheric grinding. The simulation results also reveal that the $280 \mathrm{~mm}$ aspheric surface generates fewer control vertices. The error of this fitted aspheric curve also meets general grinding requirements, thereby reducing the error $d$ to obtain additional control points. By evaluating the accuracy of the fitting curves, a NURBS fitting curve that meets accuracy requirements can be achieved by reselecting the error $d$.

3.4. Grinding Experiment Analysis. The main improvements that can be made to the large-scale aspheric grinding process are reducing the frequency of accelerations and decelerations caused by linear interpolation and improving the grinding efficiency. In this study, grinding experiments were conducted to verify the accuracy, efficiency, and overall effectiveness of the proposed algorithm. For off-axis aspheric mirrors, the proposed NURBS interpolation method was used to plan the grinding path, and finally, grinding was tested and analysed. In this paper, the grating grinding mode NC machine which has three linear axes with large-diameter workpiece is proposed to process offaxis aspheric mirrors by the NURBS interpolation method, The $X \quad Y \quad Z$ range of the grinding machine is $1000 \times 600 \times 600 \mathrm{~mm}$. In this machine, the disk-type grinding wheel for the grating grinding mode is adopted. The experimental process is to verify the effectiveness of the fine grinding stage, so the grinding wheel abrasive grain is $\mathrm{D} 15 \mathrm{~A}$, the grinding wheel radius is $130 \mathrm{~mm}$, and the grinding wheel arc radius is $70 \mathrm{~mm}$. The test equipment utilized a three-axis grinding machine with NURBS interpolation functions, including an $X Y Z$ three-axis and grinding wheel spindle axis, with a minimum step distance of $0.1 \mu \mathrm{m}$ for the linear motion axis. During the grinding process, $X Y$ axis linkage and the NURBS interpolation method were used. Because the aspheric surface in this case was an off-axis aspheric mirror, it was processed with a grating processing path. Each curve had to be fed with a set $Y$ direction step, so the $Y$-axis feed was used for the off-axis
TABLE 1: The parameters off-axis aspheric mirror.

\begin{tabular}{lc}
\hline Workpiece material & Fused silica \\
\hline Workpiece dimension $a \times b(\mathrm{~mm})$ & $280 \times 160$ \\
$K$ & -1.01 \\
$R_{x}(\mathrm{~mm})$ & 2000 \\
$c$ & 0.005 \\
\hline
\end{tabular}

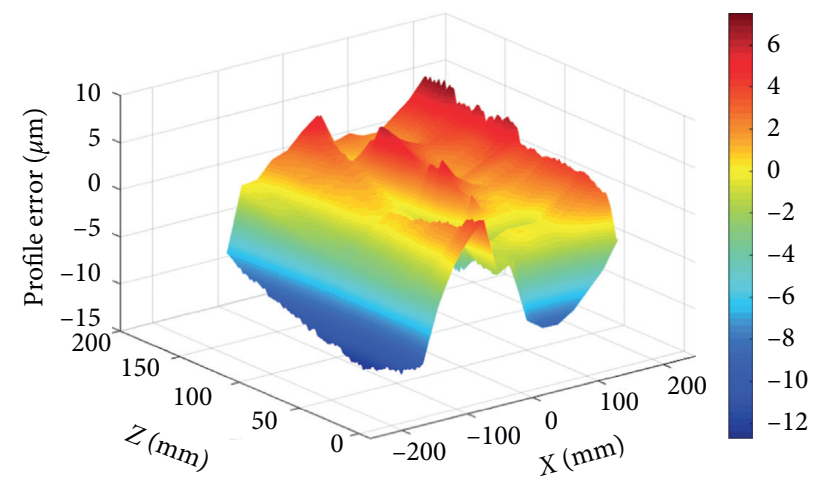

FIgURE 6: The profile error after precision grinding with linear interpolation.

aspheric surface contour processing. After grinding, a noncontact laser measurement sensor was installed at the lower end of the $Z$-axis to measure the surface contours; this provided the data for error compensation. The data could also be used for planning and analysis of the compensation interpolation path. The machine tool numerical control employs a FANUC-31i system, which can support NURBS interpolation. Here, we calculated error control points to verify the NURBS interpolation method efficiency. The control error value $d$ was set to $0.001 \mathrm{~mm}$ for analysis, and the control apex was obtained using the grinding wheel grinding point and the corresponding standard off-axis aspheric equation. We used off-axis aspheric mirrors for semiprecision grinding analysis, and the aspheric surface parameters are summarized in Table 1.

The NURBS interpolation method effectiveness was verified by conducting grinding with both linear and NURBS interpolations and comparing the results. The relevant linear interpolation parameters were calculated by the corresponding method using the off-axis aspheric surface parameters in Table 1 . Using raster processing methods, the number of NC machining program and single-direction linear interpolation points reached 29,000 and 173, respectively. The total number of CNC machining program points was multiplied by the change of the $Z$-direction processing step. The process took about 40 minutes. The surface shape after precision grinding is shown in Figure 6, and the PV value of the surface profile error was $19.3 \mu \mathrm{m}$. To improve the processing efficiency and performance of the machine tool, NURBS interpolation was implemented. The number of control vertices was determined to be 140 with this method, and the fabrication program was processed with the FANUC-31i system, which significantly reduced the number of processing program lines. The processing time was subsequently reduced to about 25 minutes, and the 


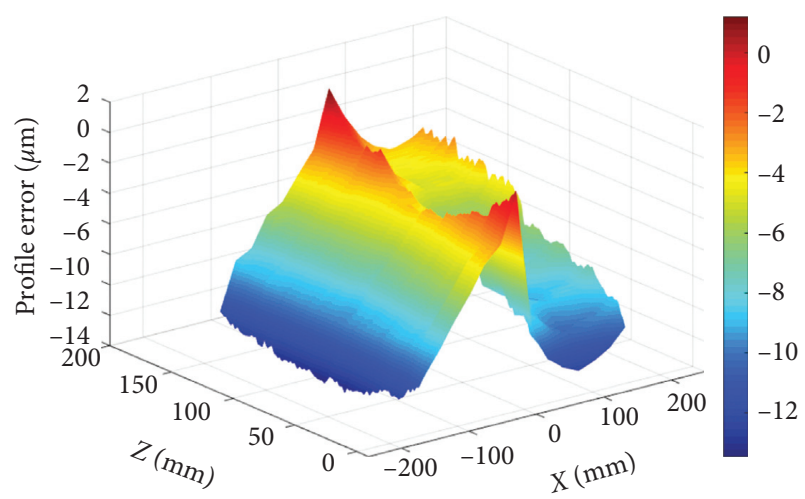

FIgURE 7: The profile error after NURBS curve interpolation grinding.

grinding efficiency was improved. The NURBS curve interpolation process reduced the continuous acceleration and deceleration of the linear axis, thus improving the surface grinding accuracy and efficiency. Figure 7 shows the error map, illustrating surface profile errors, after NURBS interpolation. The PV value was reduced to $12.6 \mu \mathrm{m}$. Obviously, NURBS interpolation scheme achieves better machining accuracy and efficiency than linear interpolation scheme. Therefore, the grinding test shows that the efficiency and surface profile accuracy have been greatly improved, and the number of acceleration and deceleration decreases, which has made progress for improving the grinding of large-diameter aspheric surfaces.

\section{Conclusions}

During processing, the grinding path and interpolation scheme used directly impact both the grinding efficiency and accuracy. Specific off-axis aspheric processing methods traditionally use linear interpolation to generate a large number of surface machining path data points; in our study, we used NURBS interpolation. The proposed scheme establishes a mathematical model for the control vertices based on the normal vector to the grinding curve, optimises the grinding path for the off-axis aspheric curve, reduces the required number of interpolation points, and fits the aspheric curve using type value points to achieve theoretical grinding accuracy. The grinding efficiency and accuracy achieved when grinding $280 \mathrm{~mm}$ off-axis aspheric mirrors were evaluated, with experimental results indicating that the NURBS interpolation scheme improved the overall machining efficiency, while the grinding surface accuracy and efficiency increased by about 30\%. Therefore, the proposed method can be employed to grind large-diameter aspheric mirrors and can provide an effective standard for high-volume high-efficiency aspheric mirror processing.

\section{Data Availability}

The data used to support the findings of this study are available from the corresponding author upon request.

\section{Conflicts of Interest}

The authors declare no conflicts of interest.

\section{Acknowledgments}

This research was funded by the National Natural Science Foundation of China (nos. 51705546 and 51975599).

\section{References}

[1] P. Shore, C. Cunningham, D. DeBra et al., "Precision engineering for astronomy and gravity science," CIRP Annals, vol. 59, no. 2, pp. 694-716, 2010.

[2] Y. Shimizu, S. Goto, J. Lee et al., "Fabrication of large-size SiC mirror with precision aspheric profile for artificial satellite," Precision Engineering, vol. 37, no. 3, pp. 640-649, 2013.

[3] R. Sarma and D. Dutta, "Tool path generation for NC grinding," International Journal of Machine Tools and Manufacture, vol. 38, no. 3, pp. 177-195, 1998.

[4] W. B. Lee, C. F. Cheung, W. M. Chiu, and T. P. Leung, "An investigation of residual form error compensation in the ultraprecision machining of aspheric surfaces," Journal of Materials Processing Technology, vol. 99, no. 1-3, pp. 129-134, 2000.

[5] P. R. Stephen, "A closed-form solution to the problem of optimal tool-path generation for sculptured surface machining on multi-axis NC machine," Mathematical and Computer Modelling, vol. 43, pp. 222-243, 2006.

[6] C.-C. A. Chen, C.-M. Chen, and J.-R. Chen, "Toolpath generation for diamond shaping of aspheric lens array," Journal of Materials Processing Technology, vol. 192-193, pp. 194-199, 2007.

[7] H. Huang, W. K. Chen, and T. Kuriyagawa, "Profile error compensation approaches for parallel nanogrinding of aspherical mould inserts," International Journal of Machine Tools and Manufacture, vol. 47, no. 15, pp. 2237-2245, 2007.

[8] Y. Hwang, T. Kuriyagawa, and S.-K. Lee, "Wheel curve generation error of aspheric microgrinding in parallel grinding method," International Journal of Machine Tools and Manufacture, vol. 46, no. 15, pp. 1929-1933, 2006.

[9] W. J. Wills-Moren and T. Wilson, "The design and manufacture of a large CNC grinding machine for off-Axis mirror segments," CIRP Annals, vol. 38, no. 1, pp. 529-532, 1989.

[10] K. Syoji, "Research on parallel grinding method of non-axisymmetric aspheric lens," Chinese Journal of Mechanical Engineering, vol. 17, no. 1, pp. 149-151, 2004.

[11] H.-T. Yau, M.-T. Lin, and M.-S. Tsai, "Real-time NURBS interpolation using FPGA for high speed motion control," Computer-Aided Design, vol. 38, no. 10, pp. 1123-1133, 2006.

[12] R. Z. Xu, L. Xie, C. X. Li, and D. S. Du, "Adaptive parametric interpolation scheme with limited acceleration and jerk values for NC machining," The International Journal of Advanced Manufacturing Technology, vol. 36, no. 3-4, pp. 343-354, 2008.

[13] S. Y. Jeong, Y. J. Choi, and P. Park, "Parametric interpolation using sampled data," Computer-Aided Design, vol. 38, no. 1, pp. 39-47, 2006.

[14] D. Du, Y. Liu, C. Yan, and C. Li, "An accurate adaptive parametric curve interpolator for NURBS curve interpolation," The International Journal of Advanced Manufacturing Technology, vol. 32, no. 9-10, pp. 999-1008, 2007.

[15] P. Comley, P. Morantz, P. Shore, and X. Tonnellier, "Grinding metre scale mirror segments for the E-ELT ground based telescope," CIRP Annals, vol. 60, no. 1, pp. 379-382, 2011. 
[16] H. Park, "A solution for NURBS modelling in aspheric lens manufacture," The International Journal of Advanced Manufacturing Technology, vol. 23, pp. 1-10, 2003.

[17] W. Sun, J. W. McBride, and M. Hill, "A new approach to characterising aspheric surfaces," Precision Engineering, vol. 34, no. 1, pp. 171-179, 2010.

[18] C. Jiang, Y. Guo, and H. Li, "Parallel grinding error for a noncoaxial nonaxisymmetric aspheric lens using a fixture with adjustable gradient," The International Journal of Advanced Manufacturing Technology, vol. 66, no. 1-4, pp. 537-545, 2013.

[19] L. Piegl and W. Tiller, The NURBS Book, Berlin: SpringerVerlag, Heidelberg, Germany, 1997. 